\title{
Implementation of persuasive design principles in mobile application development: a qualitative study
}

\author{
Nor Afifah Shafin', RD Rohmat Saedudin', Nor Hazana Abdullah ${ }^{3}$ \\ ${ }^{1}$ AxiCom Sdn. Bhd, Selangor, Malaysia \\ ${ }^{2}$ School of Industrial Engineering, Telkom University, Indonesia \\ ${ }^{3}$ Department of Technology Management, Faculty of Technology and Business Management, Universiti Tun Hussein \\ Onn Malaysia (UTHM), Malaysia
}

\begin{tabular}{l}
\hline \hline Article Info \\
\hline Article history: \\
Received Sep 15, 2019 \\
Revised Dec 15, 2019 \\
Accepted Dec 30, 2019 \\
\hline
\end{tabular}

\section{Keywords:}

Mobile application Persuasive system features Persuasive technology Persuasive technology principles Qualitative study

\begin{abstract}
Persuasive design principles (PDP) of persuasive system features framework have shown impressive results from the context of user engagement and acceptance as well as continuous usage towards the persuasive systems involved. Yet, available literatures do not thoroughly address the implementation of these design principles specifically in mobile applications and there is insufficient discussion on the impact of the principles in relation to the overall achievement of mobile applications. Hence, this research was conducted with the aim to qualitatively explore the way PDP were implemented across three different levels of mobile applications' attainment. For this study, seven semi-structured interviews were conducted with the involvement of fifteen (15) Android mobile applications in the area of utilities category. These mobile applications were then categorized into three categories which is successful, partially successful and less successful based on their numbers of mobile applications downloaded for three consecutive years. The results from the content analysis revealed that each of the PDP were implemented in many ways yet the most common applied principles are reduction, tailoring and personalization. However, the analysis also shows low numbers of implementation from the system credibility and social support category. In addition, most of the mobile applications of the successful category have implemented a lot more PDP as compared to the other two mobile application categories. The results from this study has provide significance towards developers, practitioners as well as the scholars from the contextual perspective of persuasive system framework also the practical values of the principles specifically the implementation in mobile application development.
\end{abstract}

Copyright $(2020$ Institute of Advanced Engineering and Science. All rights reserved.

\section{Corresponding Author:}

Nor Hazana Abdullah,

Department of Technology Management,

Universiti Tun Hussein Onn Malaysia (UTHM), Malaysia.

Email: hazana@uthm.edu.my

\section{INTRODUCTION}

Persuasive is one of the imperative qualities that software products such as interactive tools, websites, software systems and mobile applications should have. Furthermore, persuasive quality has been listed as the essence for great user experience other than usability (quality of software products), utility (usefulness of software products), graphic design (personality expression of software products) and functional integrity (credibility of software products) [1,2]. Although persuasive technology studies have been available since the early of 2000s and the impacts of this implementation have been known by the persuasive technology scholars, yet this domain especially the incorporation of the persuasive principles in mobile applications are still omnipresence. Furthermore, the motivation behind this study is that there are thousands of mobile applications 
release daily in the mobile application stores, yet recent statistics have shown that the quality of mobile applications have been declined hence resulting in more mobile applications being abandoned [3] or only used once $[4,5]$ and being neglected on uninstalled by the users [6]. This situation does not only affect the credibility which represents the developers, it also may even affect the revenue gained by the companies for that particular mobile application. Feng, Hoegler and Stucky [7] and Wohl, Parush, Kim, and Warren [8] described one of the major cause that lead towards this situation is the mobile application design and quality. This shows that there is the need for more researches conducted specifically on the incorporation of persuasive design principles in mobile application development. Hence, the incorporation of persuasive technology principles through the functions and features of the mobile application is hoped to improve the quality of the software products. This is because, there are numbers of empirical studies pertinent to the research of persuasive technology principles have shown promising results in getting the users to perform the action which can lead towards continuous usage and user engagement.

The significance of this study is to identify the way persuasive design principles being implemented and how these design principles may have affected the performance of the mobile applications selected for this study. In doing so, this study is able to highlight the importance of persuasive aspects in mobile application development in elevating the quality of the products developed for the users.

\section{LITERATURE REVIEW}

The studies of persuasive technology were built on multi-disciplinary domain such as humancomputer interaction and psychological behaviorism. Theories such as Information Processing Theory [9], Cognitive Consistency Theory [10], Elaboration Likelihood Model [11] and Fogg's Behavioral Model [12] are some of the theories that generally explained the persuasion process and focused on narrower area of persuasion. Furthermore, these theories also have been associated with the requirements and the nature of human-computer interaction as to elevate and enable the improvement of the overall persuasion process with the involvement of technology. To date, these theories and studies of behavioral and attitude studies have been the fundamental for the development of persuasive design principles' model and framework.

As for persuasive principles studies, there are three models and framework that have list out the principles of persuasion. These models and framework include: Influence Technique Approach [13], Functional Triad [14] and Persuasive System Features [15] which is a part from Persuasive System Design [15] framework. The Influence Technique Approach was introduced by Cialdini [16] has explained and identified the reasons for the decision made by individuals through six techniques of persuasion. These techniques include reciprocation, commitment and consistency, social proof, liking, authority and scarcity. Furthermore, this approach has been widely implemented and applied in diverse areas of studies such as marketing [17-19] and proper information delivery (communication process) as the understanding of these techniques allow the individuals to master the fundamental techniques of persuasion in the daily life. Although this theory is well-known and has been widely applied in the discussion for the proper implementation of the principles are too vague and each of the technique does not specify on the perspective of technological development. Hence, there is lacking of a structural guideline that can be referred especially by the practitioners for the implementation in mobile application development.

Next, Fogg [20] has performed much more in-depth studies on persuasion and computational products hence he has proposed a framework which is known as Functional Triad [20]. The main objective of this framework is to provide the classification of computational roles from the perspective of users that comprised with tools, media and social actors and for each of the roles, there are several persuasive principles listed to represent as well as support the core functions of that particular roles. The persuasive principles of Functional Triad are listed in Table 1. The first role of computational product known as tools play the role of increasing users' capability by reducing the effort required for the users to complete a certain action and providing a set of personalized information related to the users. Hence, the computational products that incorporated the principles which have represented certain purposes will be much more persuaded. Secondly, the media category is usually applied to provide experiences to the users in three different ways which is either through cause-and-effect, environmental and through object. Now, the role of media can be seen through the virtual reality technology as it allows the users to experience new environment without involving any harmful consequences yet they are still able to gain the experience from the situation or the environment which they have been assigned on. Lastly, social actors help to create virtual relationship in between users and the technological products. This relationship is usually created by the interactive and appealing interface design, giving encouragement or positive feedbacks or even providing familiar and understandable languages or sentences.

Although this framework has been recognized by the researchers of persuasive technology studies, there are several deficiencies that can be identified. As mentioned by Harjumaa and Oinas-kukkonen [21] also 
with the support from literature review analysis conducted in the range of 2006 to 2016 for studies conducted and published in relevant journals and conference proceedings in persuasive technology studies show that most of the principles implemented are from the tools category and there is only little number of studies which have been implemented the principles of the media and social role categories. Moreover, Fogg in his book, Persuasive Technology: Using Computers to Change What We Think and Do [12] has discussed on the need of credibility in a persuasive system. Yet, this need is not included and deliberated in the Functional Triad. In addition to that, the principles included in this framework are acceptable for the development of information system yet it does not further explain in the context of software requirement thoroughly and in more detailed.

The third model is persuasive design principles which is a part from persuasive system design; a model proposed by Oinas-Kukkonen and Harjumaa [15] that discussed and emphasized the overall development process of persuasive system. Persuasive design principles are a set of comprehensive non-functional requirements which represent the specification of content and functionality of a persuasive system. Furthermore, PSD comprised with four main categories which are primary task support (support the main task of the system), dialogue support (support the virtual relationship and communication of the system), system credibility support (create perceived credibility for the system) and social support (leveraging social influence within the users of the system). The persuasive design principles for these categories are listed in Table 1.

Table 1. The persuasive of the functional triad [17] and persuasive system design [20]

\begin{tabular}{|c|c|c|c|}
\hline Roles & Persuasive principles & Category & Persuasive Design Principles \\
\hline \multirow[t]{7}{*}{ Tools } & Reduction & Primary task support & Reduction \\
\hline & Tunneling & & Tunneling \\
\hline & Tailoring & & Tailoring \\
\hline & Suggestion & & Personalization \\
\hline & Self-monitoring & & Self-monitoring \\
\hline & Surveillance & & Simulation \\
\hline & Conditioning & & Rehearsal \\
\hline \multirow[t]{7}{*}{ Medium } & Simulated cause-and-effect & Dialogue support & Praise \\
\hline & Simulated environments & & Rewards \\
\hline & Simulated objects & & Reminders \\
\hline & & & Suggestion \\
\hline & & & Similarity \\
\hline & & & Liking \\
\hline & & & Social role \\
\hline \multirow[t]{14}{*}{ Social actor } & Physical & System credibility support & Trustworthiness \\
\hline & Psychological & & Expertise \\
\hline & Language & & Surface credibility \\
\hline & Social dynamics & & Real-world feel \\
\hline & Social roles & & Authority \\
\hline & & & Third-party endorsement \\
\hline & & & Verifiability \\
\hline & & Social support & Social learning \\
\hline & & & Social comparison \\
\hline & & & Normative influence \\
\hline & & & Social facilitation \\
\hline & & & Cooperation \\
\hline & & & Competition \\
\hline & & & Recognition \\
\hline
\end{tabular}

As shown in the Table 1, the persuasive principles of the system features were comprised with much more radical principles that emphasizes on system requirements with several new principles added as compared to Functional Triad. Also, the categories support the aspect of system and the users as PDP covers the main function of the persuasive system, ensure that there is a mutual relationship in between the users and the system by giving the feedbacks and recommendation based on users' needs, creating perceived credibility so that the users will and may trust the information as well as the organizational or the bodies involved behind the computational products and lastly giving the support needed by the users by providing a community of users that have the same intention and aims. As mentioned before, the principles of this framework suit the development of persuasive system and as mentioned by Oinas-Kukkonen and Harjumaa [15] these principles should be transformed as software requirements and system features. On top of that, there are numbers of studies that have proven that PSD are able to increase users' adherence towards system, increase self-efficacy, create technology acceptance, allow user engagement with high activation and retention rate, create emotional and visual excitement and lead to user satisfaction with positive experience towards the incorporated software products as shown in Table 2. 
Table 2. Previous studies of PDP

\begin{tabular}{ll}
\hline Persuasive technology principles benefit & Researchers \\
\hline Adherence towards system & {$[21],[22],[23],[24],[25]$} \\
Perceived self-efficacy & {$[26],[27],[28],[29],[30],[31]$} \\
Technology acceptance & {$[32],[33],[34],[35],[36],[37],[38],[39],[40]$} \\
User engagement & {$[32],[41],[42],[43],[44],[45],[46],[47],[48],[49],[50],[38]$} \\
(high activation and retention rate) & {$[51,[47],[52]$} \\
User emotional and visual excitement & {$[46],[53],[52],[54],[55],[56],[44]$} \\
User satisfaction & \\
(Positive experience) &
\end{tabular}

Therefore, in this study, PSD is selected as the best guideline to identify the persuasive system design implemented in the mobile applications selected for this study as it provides more detailed and comprehensive set of system requirement that allows the systems developed to persuade the users and eventually will creating user engagement, satisfaction and also created users' adherence towards the mobile applications. The next section of this paper will then discuss on research methodology, results and discussion as well as conclusions of this study.

\section{RESEARCH METHODOLOGY}

The research design of this research follows multiple case studies as it supports the nature of the research by allowing the researcher to gain in-depth understanding on the context of the persuasive technology principles incorporated from the developers' perspectives and experiences hence producing detailed descriptions which will then be compared to identify the differences and similarities in between the cases. For this study, three categories of mobile applications which are successful, partially successful and less successful were identified and distinguished based on the numbers of application downloaded for the past three years. Seven participants were selected among project leader, project managers and programmers as these individuals have the adequate experiences and capabilities in ensuring the data collection process was able to meet the objective of this research.

Table 3. Classification of mobile applications based on the categories

\begin{tabular}{lcc}
\hline $\begin{array}{c}\text { Categories of mobile } \\
\text { application }\end{array}$ & Mobile applications (MA) & $\begin{array}{c}\text { Number of mobile application downloaded for the } \\
\text { past three years }\end{array}$ \\
\hline Successful & $4,8,9,3,14,1$ & $10,000-100,000$ \\
Partially successful & $2,10,11$ & $1,000-10,000$ \\
Less successful & $6,15,12,7,5,13$ & $<1,000$ \\
\hline
\end{tabular}

Furthermore, qualitative technique with in-depth interviews were applied for the data collection process. As mentioned by (Gay, Mills and Airasian, [57] and Zikmund et al., [58]), in-depth interviews allow the researcher to probe the participants in gaining thorough and in-depth interpretation of information based on the participants point of view. During the interview session, a set of interview guideline was used and were changed or more questions were added depending on the flow of the conversation. This was done to support the statement given hence enabling the participants to provide detailed information of features and functions incorporated in the mobile applications developed. In addition, the interviews were conducted in Malay and English language and took approximately 90 to 110 minutes. Each of the interview sessions were tape-recorded for the process of transcribing. Moreover, content and thematic analysis were conducted. NVivo software was used to analyzed the content of the interview before the data were compared within the cases and in-between the cases.

\section{RESULT}

Provide a statement that what is expected, as stated in the "Introduction" chapter can ultimately result in "Results and Discussion" chapter, so there is compatibility. Moreover, it can also be added the prospect of the development of research results and application prospects of further studies into the next (based on result and discussion). From the analysis, it shows that there were several distinctive differences for the incorporation of persuasive system design principles among the three categories of mobile applications. As shown in Table 4, mobile applications of the successful category have incorporated a lot more persuasive principles as compared to the other two categories. 
Table 4. The incorporation of persuasive design principles across the three categories of mobile applications

\begin{tabular}{llcc}
\hline & Successful & Partially Successful & Less Successful \\
\hline Primary Task Support & Reduction & Reduction & Reduction \\
& Tunneling & Tunneling & Tailoring \\
& Tailoring & Tailoring & Personalization \\
& Personalization & Personalization & Liking \\
& Self-monitoring & Simulation & \\
Dialogue Support & Rehearsal & Rehearsal & Liking \\
& Liking & Praise & \\
& Praise & & Expertise \\
& Reminders & & \\
& Rewards & & \\
& Similarity & & \\
& Suggestion & Expertise & \\
Trustworthiness & Expertise & & \\
& Third-party endorsement & & \\
& Verifiability & & \\
& Surface credibility & & \\
& Social facilitation & & \\
& Cooperation & & \\
\hline
\end{tabular}

\subsection{The Incorporation of PDP of the Primary Task Support Category}

The primary task support category includes design principles which were mostly incorporated as to elevate the ability and motivation of the users to perform the action or use the mobile applications. From the table below, most of the persuasive design principles were incorporated in almost all of the mobile applications. Yet, several design principles such as self-monitoring, simulation and rehearsal were absent in the partially successful and less successful category.

Table 5. Numbers of persuasive design principles of the PTS incorporated across categories

\begin{tabular}{lccc}
\hline \multicolumn{1}{c}{ Persuasive design principles } & $\begin{array}{c}\text { Successful } \\
\text { (out of 6) }\end{array}$ & $\begin{array}{c}\text { Partially successful } \\
\text { (out of 3) }\end{array}$ & $\begin{array}{c}\text { Less successful } \\
\text { (out of 6) }\end{array}$ \\
\hline Reduction & 6 & 3 & 5 \\
Tunneling & 4 & 1 & 2 \\
Tailoring & 5 & 2 & 4 \\
Personalization & 6 & 2 & 3 \\
Self-monitoring & 2 & - & - \\
Simulation & 1 & 1 & - \\
Rehearsal & 1 & 1 & - \\
\hline
\end{tabular}

The principle of reduction was found to be the most incorporated primary task support principles across the three categories. The majority of the mobile applications 14 out of 15 mobile applications have applied this design principle commonly through the one-click button and the automatic geo-location detection system. Furthermore, tailoring and personalization were found in almost all of the mobile applications of the three categories as 11 out of 15 mobile applications have incorporated these two design principles. Tailoring were implemented in the form of tailored notifications, feedbacks and attributes based on users' personal preferences while personalization was identified through the way the mobile applications allowed the users to personalize the mobile applications according to their needs such as their information background, maps selection, and languages. In addition, four out of seven mobile developers have mentioned that it is imperative to allow the users to cater the information or the content of the mobile applications to their preferences as mobile application should be personalized as the mobile phones were made to be individualistic for the users.

Tunneling were identified in most of the mobile applications in the successful category (4 out of 6) while this design principle was only incorporated in one mobile application for both of partially and less successful category. This design principle was commonly implemented through the sequence of activities or actions required by the users to complete. The remaining primary task support design principles were found in far fewer mobile applications. Self-monitoring (2 out of 15), rehearsal (2 out of 15) and simulation (1 out of 15). Self-monitoring was incorporated by monitoring and reviewing users' vehicles' speed and monthly spending habit while rehearsal was identified through the mobile applications which allow the users to rehearse the activity by allowing the users to follow the required steps through real-time instructions of tutorial process. Finally, simulation was identified as the mobile application allows the users to experimenting with the elements of photography for the pictures taken. 


\subsection{The Incorporation of PDP of the Dialogue Support Category}

The dialogue support category includes design principles that help to support virtual relationship of human-computer interactions. The incorporation of persuasive design principles of this category was not comprehensive despite the benefits of the principles towards interpersonal interactions. Overall, the design principles of dialogue support category were only identified in the successful category. Furthermore, based on the Table 6, liking was the most incorporated design principles followed by reminder and similarity while social role was found to be absent in the mobile applications selected.

Table 6. Numbers of persuasive design principles of the DS incorporated across categories

\begin{tabular}{lccc}
\hline Persuasive design principles & $\begin{array}{c}\text { Successful } \\
\text { (out of 6) }\end{array}$ & $\begin{array}{c}\text { Partially successful } \\
\text { (out of 3) }\end{array}$ & $\begin{array}{c}\text { Less successful } \\
\text { (out of 6) }\end{array}$ \\
\hline Praise & 1 & 1 & - \\
Reward & 1 & - & - \\
Reminder & 3 & - & - \\
Suggestion & 1 & - & - \\
Similarity & 3 & - & - \\
Liking & 3 & 2 & 1 \\
Social role & - & - & - \\
\hline
\end{tabular}

Liking was mostly implemented in a way that the mobile applications were considered based on the aesthetical aspect such as appealing visual and information display and pleasing interface design. Furthermore, reminder was incorporated by sending alerts of users' speed limit to remind them on their driving behavior or personalized notifications to remind the users on their train schedule. Similarity were identified by the used of social cues in the mobile applications such as providing similar map interface as the Google maps and creating the same interface as the main company's website. Moreover, praise was given as an appreciation to the users through the messages sent to the users when they have completed their tickets purchased and once the users manage to get full stars on the picture captured through the simulation module. In addition, reward was given to the users in a form of virtual medal which consist of gold, silver and bronze to represent their level of achievement and to encourage them once they have made an update on the train real-time status. Finally, suggestion was identified as the application provide food articles and restaurants suggestions based on users' previous personal preferences and locations.

\subsection{The Incorporation of PDP of the System Credibility Support Category}

The system credibility support category includes design principles which will create perceived credibility and elevate the persuasive capability of the mobile applications. Nevertheless, this category was not well incorporated in the mobile applications selected for this study as most of these design principles were incorporated in the successful category. Yet, the principle of expertise was identified in one mobile application for each of the category. However, the real-world feel principle was unidentified in the fifteen mobile applications involved in this study.

Table 7. Numbers of persuasive design principles of the SCS incorporates across categories

\begin{tabular}{lccc}
\hline Persuasive design principles & $\begin{array}{c}\text { Successful } \\
\text { (out of 6) }\end{array}$ & $\begin{array}{c}\text { Partially successful } \\
\text { (out of 3) }\end{array}$ & $\begin{array}{c}\text { Less successful } \\
\text { (out of 6) }\end{array}$ \\
\hline Trustworthiness & 4 & - & - \\
Expertise & 1 & 1 & 1 \\
Surface credibility & 2 & - & - \\
Real-world feel & - & - & - \\
Authority & 4 & - & - \\
Third-party endorsement & 4 & - & - \\
Verifiability & 2 & - & - \\
\hline
\end{tabular}

Trustworthiness design principle was identified through the mobile application that delivered truthful and accurate information. The credibility and trustworthiness of the information delivered was associated with the involvement of expertise such as the experience in the area of expertise, authority or having the endorsement from a third reliable party such as the involvement of the bodies of government or private sectors. The involvement of these credible organizations will create perceived credibility to the users. The incorporation of these design principles was identified as these applications were developed based on the sources from two reputed and well-known halal organizations in Malaysia, developed by an individual that has more than five 
years' experience in photography and lastly developed based on the information available on the Malaysia's government website.

Surface credibility design principle was identified based on the resemblance of the organization which the mobile application representing. As an example, the two mobile applications identified with surface credibility were aesthetically design based on the corporate identity also the main website of the organization represented. Finally, verifiability is the ability for the users to verify the accuracy of the information delivered. Two of the mobile applications have provided a link and a section that contains contact number of organization involved and the organization's website for the users to validate the information presented as well as the credibility of the mobile application.

\subsection{The Incorporation of PDP of the Social Support Category}

The social support category includes design principles which help to elevate social support among the mobile application's users. Based on the Table 8, the principles from this category were mostly have not been incorporated in the mobile applications. From the analysis, only social facilitation and cooperation were identified in two out of fifteen mobile applications.

Table 8. Numbers of persuasive design principles of the SSC incorporated across categories

\begin{tabular}{lccc}
\hline Persuasive design principles & $\begin{array}{c}\text { Successful } \\
\text { (out of 6) }\end{array}$ & $\begin{array}{c}\text { Partially successful } \\
\text { (out of 3) }\end{array}$ & $\begin{array}{c}\text { Less successful } \\
\text { (out of 6) }\end{array}$ \\
\hline Social learning & - & - & - \\
Social comparison & - & - & - \\
Normative influence & - & - & - \\
Social facilitation & 2 & - & - \\
Cooperation & 2 & - & - \\
Competition & - & - & - \\
Recognition & - & - & - \\
\hline
\end{tabular}

In both of the mobile applications, social facilitations were implemented by providing a platform for information sharing among the users. In addition, cooperation was implemented which allow the users to encourage among themselves for much more engaging activities by providing real-time status updates or reports on the current situations.

\section{CONCLUSION}

In conclusion, it is obviously noticed that the incorporation of persuasive design principles has shown to be associated with the achievement of mobile applications as higher numbers of persuasive features have led to higher number of mobile applications downloaded. This paper aims to identify the way persuasive design principles implemented in three mobile applications' attainment and also to see the correlation in between the implementation of these persuasive principles towards the achievement of the selected mobile applications. To achieve these objective, 15 Android mobile applications were selected of the utilities category and semi-structured interviews were conducted with seven mobile developers. Furthermore, probing technique was uses during the interview to gain thorough description on the way PDPs were implemented in the mobile application developed.

The content analysis from the interviews revealed that the principles of the primary task support category is the most frequently implemented principles with reduction, tailoring and personalization being the most applied as these design principles can almost be identified across the three categories of mobile applications. Overall, reduction was implemented by creating a one-click button and automated geo-location detection system and tailoring and personalization were identified by the mobile applications that provide tailored content or information that is curated based on users' preferences which is usually identified by the users after they have installed the mobile application. Furthermore, based on the cross-cases analysis conducted have showed that the mobile applications of the successful category have implemented almost $68 \%$ of the persuasive design principles compared to the partially successful category which is $32 \%$ and less successful which is only nearly $21 \%$. However, as stated by Oinas-Kukkonen and Harjumaa [21], the incorporation of these design principles should be applied based on the requirement of the system. Yet, the implementation of two or more principles for a single function for example reduction-tailoring-personalization allow to create much more superior persuasion towards the users.

However, this study also revealed several shortcomings which should be given the attention on for the improvement of further studies. Based on the analysis, there are only selected design principles which have 
been incorporated of the system credibility and social support categories. Both of these categories have said to be giving much more persuasion effect for creating perceived credibility on the software products also creating a community within the users of the mobile application. Hence, the incorporation of these design principles is imperative for a better quality of mobile applications.

As a conclusion, persuasive design principles have provided the adequate application guideline which comprised the non-functional requirements along with the need of the mobile applications as well as the users based on the four categories. Nevertheless, these principles should be highlighted and emphasized by the research community also for the developers to improve and provide the knowledge of the guideline and the way persuasive design principles should be applied in the mobile application development.

\section{ACKNOWLEDGEMENTS}

This research is funded under FRGS Grant No. U107 under UTHM.

\section{REFERENCES}

[1] D. J. Mayhew, "Usability + Persuasiveness + Graphic Design = eCommerce User Experience," in The HumanComputer Interaction Handbook, 3rd ed. Springer US, 2009, pp. 1181-1194.

[2] E. Marache-Francisco, C. Brangier, "The Gamification Experience: UXD with a Gamification Background," in Gamification: Concepts, Methodologies, Tools and Applications, USA: Information Resources Management Associations, 2015.

[3] H. Levenson, "7 Common Reasons Users are Abandoning your App," 2016. [Online]. Available: https://www.webanalyticsworld.net/2016/08/why-users-are-abandoning-your-mobile-app.html. [Accessed: 03-Jan2017].

[4] J. Pramis, "Are you a rarity? Only 16 percent of people will try out an app more than twice," 2013. [Online]. Available: https://www.digitaltrends.com/mobile/16-percent-of-mobile-userstry-out-a-buggy-app-more-than-twice/. [Accessed: 01-Jan-2017].

[5] J. Dye, "77 percent of users never use an app again 72 hours after installing," 2016. [Online]. Available: http://www.androidauthority.com/77-percent-users-dont-use-an-app-after-three-days-678107/. [Accessed: 19-Jan2017].

[6] M. Böhmer, B. Hecht, J. Schöning, A. Krüger, and G. Bauer, "Falling asleep with Angry Birds, Facebook and KindleA Large Scale Study on Mobile Application Usage, ” Proc. 13th Int. Conf. Hum. Comput. Interact. with Mob. Devices Serv. - MobileHCI '11, p. 47, 2011.

[7] H. Feng, T. Hoegler, and W. Stucky, "Exploring the critical success factors for mobile commerce," Mob. Business, 2006. ICMB'06. Int. Conf., 2006.

[8] M. J. A. Wohl, A. Parush, H. A. S. Kim, and K. Warren, "Building it better: Applying human-computer interaction and persuasive system design principles to a monetary limit tool improves responsible gambling," Comput. Human Behav. vol. 37, pp. 124-132, 2014.

[9] W. J. McGuire, "Persuasion," in Communication, language, and meaning Psychological perspectives, Editors. Basic Books, 1973, pp. 242-255.

[10] C. Fraser, B. Burchell, and D. Hay, "Introducing social psychology," Cambridge: Polity, 2001.

[11] R. E. Petty and J. . Cacioppo, "Communication and Persuasion: Central and Peripheral Routes to Attitude Change," New York: Springer, 1986.

[12] B. J. Fogg, "Computers as Persuasive Social Actors," in Persuasive Technology: Using Computers to Change What We Think and Do. Elsevier Science and Technology Books, 2003, pp. 89-120.

[13] R. Guadagno and R. Cialdini, "Online persuasion and compliance: social influence on the Internet and beyond," in The Social Net: Understanding human behavior in cyberspace, Oxford: Eds. Oxford University Press, 2005.

[14] B. J. Fogg, "Persuasive Technology: Using Computers to Change What We Think and Do," Ubiquity, 2002.

[15] H. Oinas-kukkonen and M. Harjumaa, "A Systematic Framework for Designing and Evaluating Persuasive Systems," International Conference on Persuasive Technology. pp. 164-176, 2008.

[16] R. Cialdini, "Pre-Suasion: A Revolutionary Way to Influence and Persuade," Simon and Schuster, 2016.

[17] T. R. Bacon, "Elements of Influence: The Art of Getting Others to Follow Your Lead. AMACOM," 2012.

[18] C. Warren, S. Becken, and A. Coghlan, "Using persuasive communication to co-create behavioural change- engaging with guests to save resources at tourist accommodation facilities," J. Sustain. Tour., vol. 25, no. 7, 2016.

[19] M. Harjumaa and S. Muuraiskangas, "Building Persuasiveness into Information Systems," Electronic Journal of Information Systems Evaluation, vol. 17, no. 1, pp. 23-35, 2014.

[20] B. J. Fogg, J. Marshall, O. Laraki, A. Osipovich, C. Varma, N. Fang, J. Paul, A. Rangnekar, J. Shon, P. Swani, M. Treinen, and C. Hall, "What Makes Web Sites Credible? A Report on a Large Quantitative Study CHI 2001," Sigchi’01, pp. 61-68, 2001.

[21] H. Oinas-kukkonen and M. Harjumaa, "A Systematic Framework for Designing and Evaluating Persuasive Systems," International Conference on Persuasive Technology. pp.164-176, 2008.

[22] R. Neff and J. Fry, "Periodic prompts and reminders in health promotion and health behavior interventions: systematic review, ” J. Med. Internet Res., vol. 11, no. 2, p. e16, 2009. 
[23] S. M. Kelders, E. T. Bohlmeijer, W. T. M. Pots, and J. E. W. C. van Gemert-Pijnen, "Comparing human and automated support for depression: Fractional factorial randomized controlled trial," Behav. Res. Ther., vol. 72, pp. 72-80, 2015.

[24] S. Munson and S. Consolvo, "Exploring Goal-setting, Rewards, Self-monitoring, and Sharing to Motivate Physical Activity,” Proc. 6th Int. Conf. Pervasive Comput. Technol. Healthc., pp. 25-32, 2012.

[25] F. Drozd, T. Lehto, and H. Oinas-kukkonen, "Exploring Perceived Persuasiveness of a Behavior Change Support System : A Structural Model, ” Int. Conf. Persuas. Technol., pp. 157-168, 2012.

[26] S. M. Kelders, R. N. Nok, H. C. Ossebaard, and J. E. Van Gemert-Pijnen, "Persuasive system design does matter: A systematic review of adherence to web-based interventions," J. Med. Internet Res., vol. 14, no. 6, p. e152, 2012.

[27] A. Bandura, "Social cognitive theory: An agentic perspective," Annu. Rev. Psychol., vol. 52, no. 1, pp. 1-26, 2001.

[28] S. M. Noar, C. N. Benac, and M. S. Harris, "Does tailoring matter? Meta-analytic review of tailored print health behavior change interventions," Psychol. Bull., vol. 133, no. 4, pp. 673-693, 2007.

[29] I. Wiafe, "A framework for analysing, designing and evaluating persuasive technologies," Ghana Institute of Management and Public Administration, 2012.

[30] J. Mumm and B. Mutlu, "Designing motivational agents: The role of praise, social comparison, and embodiment in computer feedback," Comput. Human Behav, vol. 27, no. 5, pp. 1643-1650, 2011.

[31] B. Shneiderman, "Designing the user interface: strategies for effective human-computer interaction," Pearson Ed. 2010.

[32] A. Stibe and H. Oinas-Kukkonen, "Designing Persuasive Systems for User Engagement in Collaborative Interaction," Twenty Second Eur. Conf. Inf. Syst., pp. 1-17, 2014.

[33] K. Ohk, S. Park, and J. Hong, "The Influence of Perceived Usefulness, Perceived Ease of Use , Interactivity , and Ease of Navigation on Satisfaction in Mobile Application," Advanced Science and Technology Letters vol. 84, pp. 8892, 2015.

[34] M. Shams, D. Shojaeizadeh, R. Majdzadeh, A. Rashidian, and A. Montazeri, "Taxi drivers' views on risky driving behavior in Tehran: A qualitative study using a social marketing approach,” Accid. Anal. Prev., vol. 43, no. 3, pp. 646-651, 2011.

[35] M. Kaptein, “Adaptive Persuasive Messages In An E-Commerce Setting: The Use Of Persuasion Profiles," ECIS 2011 Proc., p. Paper 183, 2011.

[36] C. N. Wathen and J. Burkell, "Believe it or not: Factors influencing credibility on the Web," J. Am. Soc. Inf. Sci. Technol., vol. 53, no. 2, pp. 134-144, 2002.

[37] J. Masthoff and J. Vassileva, “Tutorial on Personalization for Behaviour Change,” Proc. 20th Int. Conf. Intell. User Interfaces - IUI '15, vol. 2015-Janua, pp. 439-442, 2015.

[38] R. Orji, “Design for Behaviour Change: A Model Driven Approach for Tailoring Persuasive Technology,” 2014.

[39] E. Kaasinen, "User acceptance of mobile services-value, ease of use, trust and ease of adoption," Vtt Publ., vol. 566, 2005.

[40] L. Gamberini, A. Spagnolli, N. Corradi, G. Jacucci, G. Tusa, T. Mikkola, L. Zamboni, and E. Hoggan, "Tailoring feedback to users' actions in a persuasive game for household electricity conservation," Lect. Notes Comput. Sci. including Subser. Lect. Notes Artif. Intell. Lect. Notes Bioinformatic, vol. 7284 LNCS, pp. 100-111, 2012.

[41] J. Hamari, "Computers in Human Behavior Do badges increase user activity? A field experiment on the effects of gamification," Comput. Human Behav., pp. 1-10, 2015.

[42] P. Mayer, "Empirical Investigations on User Perception and the Effectiveness of Persuasive Technologies," Verdi.Unisg.Ch, no. 4028, p. 186, 2012.

[43] N. D. Lane, E. Miluzzo, H. Lu, D. Peebles, T. Choudhury, and A. T. Campbell, "A survey of mobile phone sensing," IEEE Commun. Mag., vol. 48, no. 9, pp. 140-150, 2010.

[44] Kaptein, M., De Ruyter, B., Markopoulos, P., Aarts, E“Adaptive Persuasive Systems : A Study of Tailored Persuasive Text Messages to Reduce Snacking”. ACM Transactions on Interactive Intelligent Systems, Vol. 2, No. 2, 2012.

[45] T. Lehto, H. Oinas-Kukkonen, and D. Filip, "Factors Affecting Perceived Persuasiveness of a Behavior Change Support System," Thirty Third International Conference on Information Systems, pp. 1-15, 2012.

[46] M. M. Alhammad and S. R. Gulliver, "Context relevant persuasive interaction and design: Consideration of human factors influencing $\{B 2 C\}$ persuasive interaction,” 35th Int. Conf. Inf. Technol. Interfaces, \{ITI\} 2013, pp. 161-166, 2013.

[47] T.-R. Chang, E. Kaasinen, and K. Kaipainen, “What Influences Users' Decisions to Take Apps into Use?: A Framework for Evaluating Persuasive and Engaging Design in Mobile Apps for Well-being," Int. Conf. Mob. Ubiquitous Multimed., p. 2:1-2:10, 2012.

[48] M. Sohn and J. Lee, "UP health: Ubiquitously persuasive health promotion with an instant messaging system," 25th SIGCHI Conf. Hum. Factors Comput. Syst. 2007, CHI 2007, pp. 2663-2668, 2007.

[49] T. Alahäivälä, "Software Design of a Health BCSS : Case Onnikka," 2013.

[50] L. Little, E. Sillence, and A. Joinson, "Behavior Change Research and Theory: Psychological and Technological Perspectives," Academic Press, 2016.

[51] A. Jawdat, Q. Obeidat, and A. Aljanaby, “On The Design of User Experience Based Persuasive Systems, ” Comput. Inf. Sci., vol. 4, no. 4, pp. 90-99, 2011.

[52] L. Dennison, L. Morrison, G. Conway, and L. Yardley, "Opportunities and challenges for smartphone applications in supporting health behavior change: qualitative study.," J. Med. Internet Res., vol. 15, no. 4, pp. 1-12, 2013.

[53] L. M. Ritterband, F. P. Thorndike, D. J. Cox, B. P. Kovatchev, and L. A. Gonder-Frederick, "A behavior change model for internet interventions," Ann. Behav. Med., vol. 38, no. 1, pp. 18-27, 2009.

[54] H. Oinas-Kukkonen and V. Kurkela, "Developing successful mobile applications," Reason, vol. 394, p. $54,2003$. 
[55] S. Langrial, H. Oinas-Kukkonen, and S. Wang, "Design of a Web-Based Information System for Sleep DeprivationA Trial Study Design of a Web-Based Information System,” Int. Conf. Well-Being Inf. Soc., no. August, pp. 41-51, 2012.

[56] S. Langrial and H. Oinas-kukkonen, “Less Fizzy Drinks : A Multi-method Study of Persuasive Reminders, ” Int. Conf. Persuas. Technol., pp. 256-261, 2012.

[57] L. R. Gay, G. E. Mills, and P. W. Airasian, "Educational Research Competencies for Analysis and Applications," 9th editio. Columbus, Ohio: Pearson, 2009.

[58] W. G. Zikmund, B. J. Babin, J. C. Carr, and M. Griffin, "Qualitative Research Tools," in Business Research Method, 9th ed., Canada: Nelson Education, Ltd., 2013, pp. 131-158.

\section{BIOGRAPHIES OF AUTHORS}

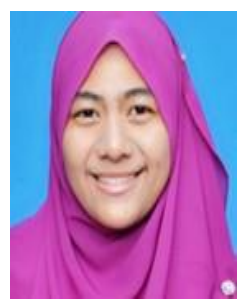

Nor Afifah Shafin had her degree in Information Technology and Master in Technology Management from Universiti Tun Hussein Onn Malaysia. She is currently working as Senior Developer at AxiCom Sdn Bhd.

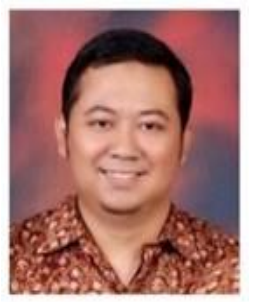

He is a senior lecturer at Study Program of Information System , School of Industrial Engineering, Telkom University, Indonesia. He is a PhD Student in Faculty of Computer Science and Information Technology at since 2015. The areas of his research are Telecommunication Information System, Soft Computing, Data Mining, and Optimization System.

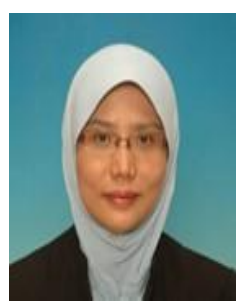

Nor Hazana Abdullah is an Associate Professor, and Deputy Dean of Research, Development and Publication at Universiti Tun Hussien Onn Malaysia. She earned her Bsc. in Psychology from Indiana University of Bloomington, USA, Master from Universiti Teknologi Malaysia and PhD from Universiti Tun Hussein Onn Malaysia. Her research focuses on human behaviors. 